\section{An Application of Embedology to Spatio-Temporal Pattern Recognition}

\author{
JAMES R. STRIGHT \\ STEVEN K. ROGERS \\ DENNIS W. QUINN \\ KENNETH H. FIELDING \\ Air Force Institute of Technology
}

The theory of embedded time series is shown applicable for determining a reasonable lower bound on the length of test sequence required for accurate classification of moving objects. Sequentially recorded feature vectors of a moving object form a training trajectory in feature space. Each of the sequences of feature vector components is a time series, and under certain conditions, each of these time series has approximately the same fractal dimension. The embedding theorem may be applied to this fractal dimension to establish a sufficient number of observations to determine the feature space trajectory of the object. It is argued that this number is a reasonable lower bound on test sequence length for use in object classification. Experiments with data corresponding to five military vehicles (observed following a projected Lorenz trajectory on a viewing sphere) show that this bound is indeed adequate.

Manuscript received May 19, 1994.

IEEE Log No. T-AES/32/2/03457.

Authors' addresses: J. R. Stright, S. K. Rogers, K. H. Fielding, Department of Electrical and Computer Engineering, Air Force Institute of Technology, 2950 P St., Wright-Patterson Air Force Base, OH 45433-7765; D. W. Quinn, Department of Mathematics and Statistics, 2950 P St., Wright-Patterson Air Force Base, OH 45433-7765.

U.S. Government work. Not protected by U.S. copyright.

$\overline{0018-9251 / 96 / \$ 10.00 ~(c) ~} 1996$ IEEE

\section{INTRODUCTION}

Fielding has recently implemented a spatio-temporal classifier based on the Hidden Markov Model technique [9] that identifies 3D objects in 2D image sequences $[3,5,4,2]$. A "viewer centered" approach is adopted placing the object of interest in the center of a transparent sphere [11]. Sequences of views are created by traversing a portion of the surface of the sphere. (The traversed portion of the surface is called the viewing quadrant, although it doesn't quite subtend a full quadrant of the sphere.) Fielding considered a five class problem of identifying the military land vehicles shown in Fig. 1. In one form of the classifier, a single 3000 frame image sequence is generated for each object, viewed from a shared random traversal of the viewing quadrant. The Hidden Markov Model for each class is trained using the sequence of feature vectors extracted from each image in the trajectory. The classifier performance is then judged using 200 short test sequences which are typically trajectories near, but not contained within, the training trajectory.
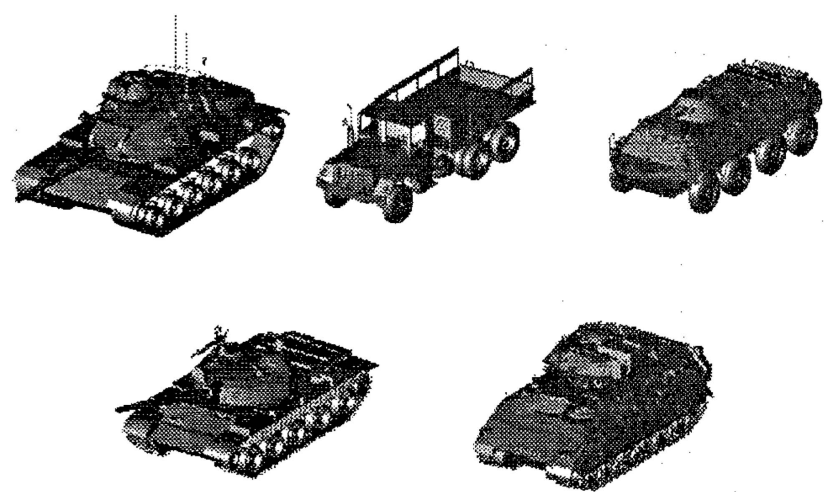

Fig. 1. Classified Objects: M60 tank, M35 truck, BTR60 APC, T62 tank, and M2 IFV.

One might wonder how best to assemble training sequences. Perhaps a region-filling curve composed of segments found most typical of actual vehicle movements (derived, for example, from observations made during training exercises) might most accurately represent real-world scenarios. Even if it is practical, however, to train with sequences containing subsequences near every possible test sequence, some critical questions have to be answered. How close is "near?" What minimum length test sequence should be used?

The problem of constructing an optimal training trajectory is not addressed here. Instead, simplifying assumptions are made on the vehicle-viewer orientations to produce training trajectories on the viewing sphere, and the fractal dimensions of the derived feature vector trajectories are then exploited 


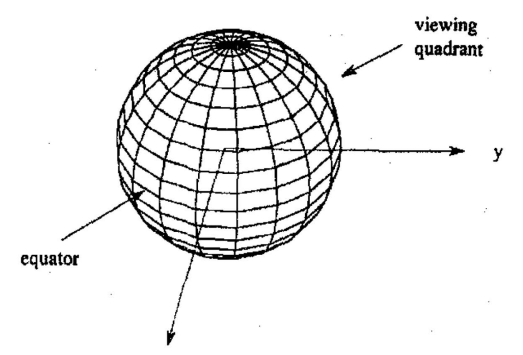

Fig. 2. Viewing sphere.

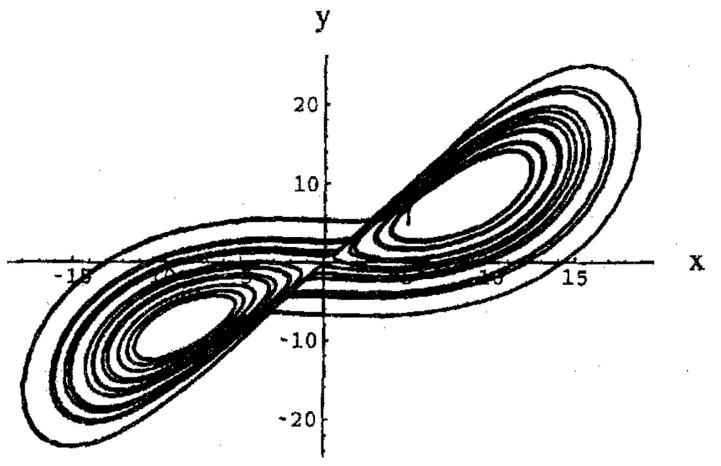

Fig. 3. Lorenz trajectory.

to infer a sufficient lower bound for test sequence lengths.

This procedure was performed using feature vectors consisting of Fourier magnitude components, and the test sequence length lower bound so determined was found adequate for classification using a Hidden Markov Model classifier. A nearest neighbor classifier was also constructed and found adequate using the same lower bound. The nearest neighbor classifier is described and its classification performance analyzed.

\section{A LORENZ VIEWING SPHERE TRAVERSAL STRATEGY}

Consider a scaled Lorenz attractor [8, p. 136] confined within 3-space to the viewing quadrant of interest, where the stationary object is centered at the origin and the $x$ and $y$ axes determine the equatorial plane; see Fig. 2. Let $\mathrm{P}$ denote the projection of the confined attractor onto the viewing quadrant surface (it does not extend to the edges of the surface, and leaves large portions of the viewing quadrant unexplored). An approximation to $P$ was derived from a solution to the Lorenz equations with the initial condition $\left(x_{0}=5, y_{0}=5, z_{0}=5\right)$; its $(x, y)$-projection is illustrated in Fig. 3.

"Snapshots" are taken of the viewed object at each point on $P$ and features are extracted from each snapshot. The features used in this work are essentially the magnitudes of the 28 dominant two-dimensional spatial Fourier coefficients associated with the views. Thus each view is condensed to a 28-tuple of real

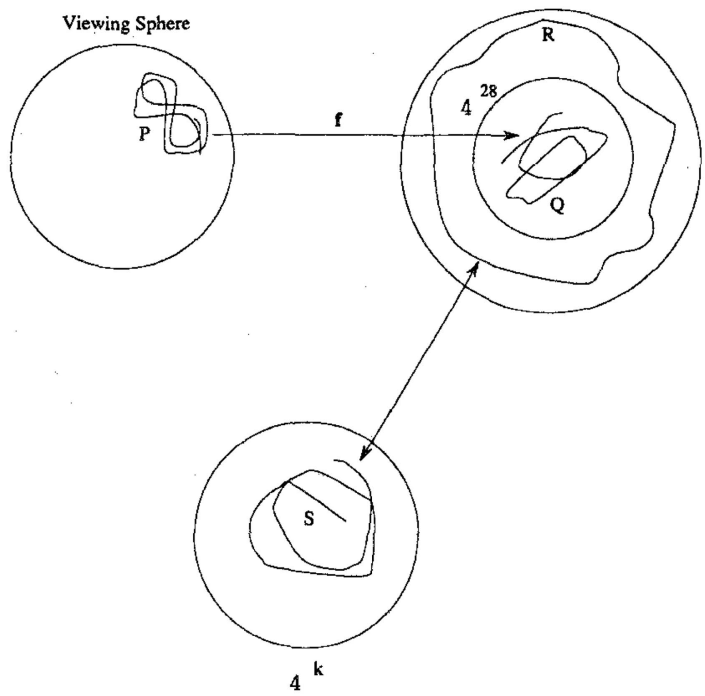

Fig. 4. Embedding time series derived from views along trajectory $P$.

numbers. A training sequence then consists of a finite number of such 28-tuples, and a test sequence consists of a smaller number of 28-tuples derived from viewing trajectories oriented near the viewing trajectory used during training. Fig. 4 illustrates conceptually a training sequence $Q$ in $\Re^{28}$, as derived from the viewing traversal $P$ via the mapping $\mathbf{f}$.

Depending on the choice of features, mappings from the viewing trajectory into feature space may be continuous in each of their components and therefore continuous themselves. Empirical evidence suggests this is a reasonable assumption for Fourier magnitude components, so that the mapping $\mathbf{f}$ of Fig. 4 may be considered continuous. Fig. 5 shows in gray-scale the fourteenth Fourier component values above the flattened viewing quadrant for each of five classes of military land vehicle: M60 tank, M35 truck, BTR60 armored personnel carrier, T62 tank, and M2 infantry fighting vehicle, as labeled. The darker is the gray in these images, the higher is the component value (with white being zero). Notice the rarity of sharp discontinuities; perhaps this is due to the complexity of these vehicles, i.e., the large number of parts with both sharp and smooth edges, no single one of which is dominant. In any case, the distributions of fourteenth component values generated from the Lorenz viewing path appear rather continuous. The fourteenth component values seemed typical in this respect to other components examined.

\section{EMBEDDING A FEATURE SPACE TRAJECTORY}

The trajectory $Q$ is an image of the physical process of viewing sphere traversal; changes along $Q$ depend upon the current position on $Q$, which is the image under $\mathbf{f}$ of a position on $P$. It is reasonable to assume that $Q$ is the solution trajectory of a system 


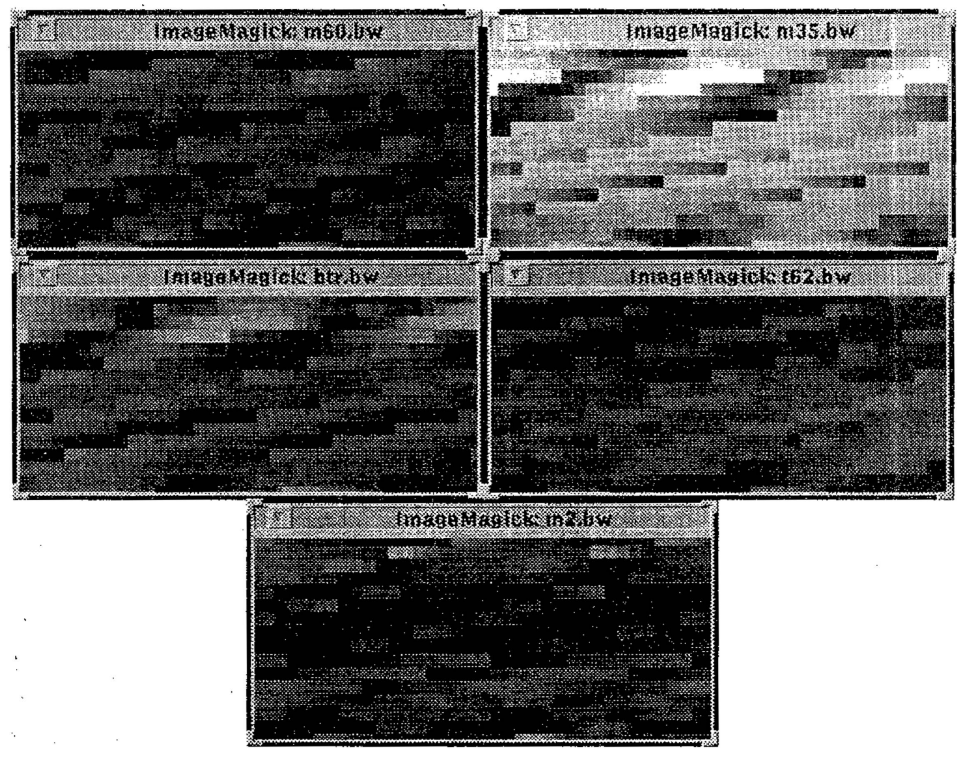

Fig. 5. Fourteenth Fourier components of 5 military vehicles.

of differential equations in $\Re^{28}$, or the projection of a solution trajectory $R$ in a higher dimensional space (as the two-dimensional Lorenz curve illustrated in Fig. 3 is the projection of a three-dimensional solution trajectory). This requires that $Q$ be continuous; but it has been argued that the mapping $\mathbf{f}: P \rightarrow Q$ is continuous, and since $P$ is continuous, $Q$ also must be continuous [1, p. 79].

Tim Sauer's Fractal Coordinate Embedding Prevalence Theorem [10,13] may now be applied to the set $R$, or rather to the components of $R$, hence to the components of its projection $Q$. This theorem holds that, under quite reasonable conditions on the dynamical system which produced $R$, a delay coordinate embedding of $R$ into $\Re^{k}$ is a diffeomorphism, as long as $k$ is greater than twice the box counting dimension $d$ of $R$.

This box counting dimension may be estimated as the correlation dimension of the time series determined by any one of the components of $R$ (hence also by any one of the components of $Q$ ). A time series $x_{1}, x_{2}, x_{3}, \ldots$ consists of a sequence of real numbers, hence there is little a priori geometric intuition behind the notion of box counting dimension of a time series. Suppose, however, that the time series consists of samples equally spaced in time and is delay coordinate embedded into a space of dimension higher than one, in the following manner. Fix some small integer $n \geq 2$. Form from the time series a set of points $\mathbf{x}_{i}, i=1,2, \ldots$ in $\Re^{n}$ by taking successive $n$-tuples from the sequence $x_{1}, x_{2}, x_{3}, \ldots$ That is,

$$
\begin{aligned}
& \mathbf{x}_{1}=\left(x_{1}, x_{2}, \ldots, x_{n}\right) \\
& \mathbf{x}_{2}=\left(x_{2}, x_{3}, \ldots, x_{n+1}\right) \\
& \mathbf{x}_{3}=\left(x_{3}, x_{4}, \ldots, x_{n+2}\right)
\end{aligned}
$$

Provided $n>2 d$, the box counting dimension of the resulting set of points $\mathbf{x}_{i}$ could be calculated directly (albeit awkwardly), and it would equal the box counting dimension of $R$. The more efficient approach of Grassberger and Procaccia [6] also uses such delay coordinate embeddings to calculate an approximately equal quantity called the correlation dimension.

Having established $d$, the delay coordinate embedding of $R$ into $\Re^{k}$ (via a component of $Q$ ) is a diffeomorphism, provided that $k>2 d$. The diffeomorphic image $S$ of $R$ is illustrated in Fig. 4.

Any $k$ consecutive values of a component of $Q$ determine a single point on $S$. Consider now the relationship between $Q$ and $S$. The fundamental theorem of differential equations assures that any point on $R$ determines all of $R[7, \mathrm{p} .162]$. Since $S$ and $R$ are diffeomorphic, any single point on $S$ not only determines all of $S$, but its image in $R$ determines all of $R$. Thus the diffeomorphic image of the point on $S$ traces the trajectory $Q$. Therefore any $k$ consecutive views of the object completely describe the trajectory $Q$, which is called the "object evolution" (or training evolution) of the viewed object, to account for its temporal as well as spatial nature.

The number $k$ has the following significance for object classification. Suppose there are five objects subject to classification, with training evolutions $C^{1}$ through $C^{5}$ in $\Re^{28}$ derived from identical traversals $P$ of the viewing sphere. Suppose that no pairs of evolutions $C^{i}$ and $C^{j}$ overlap in $k$ or more consecutive points. If a test evolution in $\Re^{28}$ is derived from $k$ or more views of one of the five objects taken along the trajectory $P$, then the test evolution will uniquely determine the entire training evolution of the viewed object.

The evolutions $C^{1}$ through $C^{5}$ can be screened to determine the maximum amount of overlap, and 


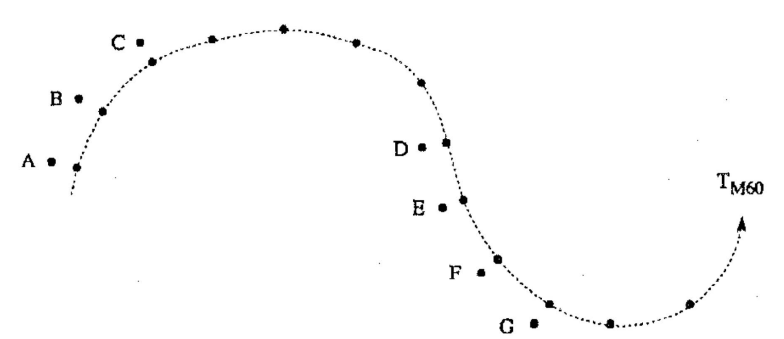

Fig. 6. Portions of trajectories in $\Re^{28}$.

the number $k$ may have to be increased to exceed that amount. More problematic, however, is the fact that test sequences cannot be expected to come from traversals of $P$; at best, they can only be assumed to come from traversals near $P$. Fig. 6 illustrates the uncertainty inherent in spatio-temporal classification. The curve marked $T_{\mathrm{M} 60}$ represents a portion of a training trajectory in $\Re^{28}$ corresponding to a particular viewed object M60. The points $A, B$, and $C$ represent points in $\Re^{28}$ derived from consecutive testing views of the object taken near, but not on, the training trajectory; so do the points $D, E, F$, and $G$, obtained during a separate test. Suppose that all the points $A$ through $G$ are at the same small Euclidean distance from points on $T_{\mathrm{M} 60}$, and that none of the other testing points are as close. How likely is it that the object viewed during the test traversal which yielded the points $A, B$, and $C$ is an M60? Less likely, intuitively, than the object viewed during the test traversal which yielded the points $D, E, F$, and $G$, because the latter testing trajectory tracked the training trajectory over a longer period of time. Similarly, greater confidence would attach to five consecutive close points than to four. The significance of the number $k$ is that if $k$ consecutive testing points coincide exactly with $k$ consecutive points on $T_{\mathrm{M} 60}$, and the testing sequence was obtained following the same viewing quadrant traversal as the one which yielded $T_{\mathrm{M} 60}$, then the testing trajectory and $T_{\mathrm{M} 60}$ must be identical. For testing trajectories not directly on $T_{\mathrm{M} 60}, k+1$ or more consecutive near points may reasonably be interpreted as stronger evidence of an M60 than $k$ consecutive near points. Nevertheless, lacking specification of allowable test sequences, the embedology-derived number $k$ may be a reasonable compromise test sequence length to use in practice.

\section{A NEAREST NEIGHBOR CLASSIFIER}

A nearest neighbor classifier was implemented to determine if test sequences of length $k$ were adequate for accurate vehicle classification. Given a test sequence, this technique for determining its class is to match its point-by-point nearness to all sequences of the same length contained in all the training data. The class of the training sequence providing the best fit is declared the most likely class. Consider, for example,

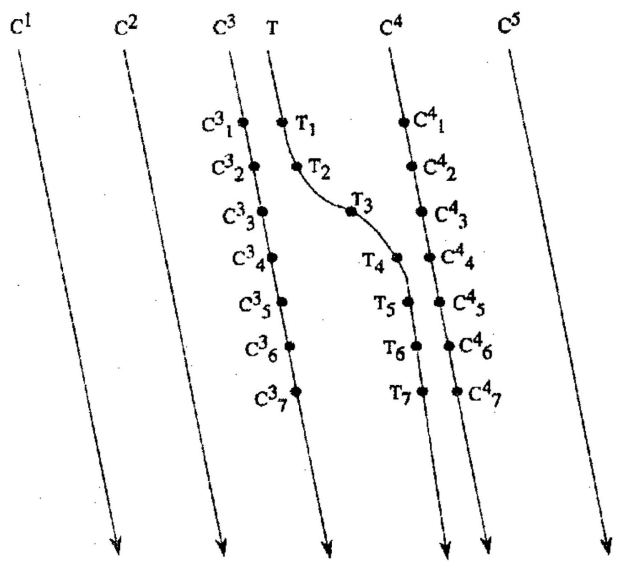

Fig. 7. Trajectories in $\Re^{28}$.

hypothetical portions of five training sequences $C^{i}$ as shown in Fig. 7. The sampled test sequence in Fig. 7 consists of seven points, and its trajectory through phase space is labeled $T$. The sum of the squared Euclidean distances from the seven points $T_{1}$ through $T_{7}$ to the points $C_{1}^{3}$ through $C_{7}^{3}$, respectively, is greater than the sum of the squared distances from $T_{1}$ through $T_{7}$ to the points $C_{1}^{4}$ through $C_{7}^{4}$, respectively. Indeed, there is no sequence of seven points in any class which is closer to the test sequence than the seven points $C_{1}^{4}$ through $C_{7}^{4}$. The test sequence is therefore identified as class four.

An equivalent statement of the classification procedure may be found by imagining the test sequence a single point $T$ in $7 \times 28=196$ space. That is, concatenate the 28 components of each of the 7 points into a single vector $T$ of dimension 196. Starting with each point (prior to the final six) in all the training classes, form 196-tuples from the concatenated components of the starting point and the six points following it. The class of the point in 196-space nearest $T$ in Euclidean distance is declared the class to which $T$ belongs. This was the approach taken in developing the nearest neighbor algorithm used in this research.

\section{EXPERIMENTAL RESULTS}

The Lorenz traversal described earlier was applied to obtain training sequences of views of each of the five military target classes. After the confined Lorenz attractor was generated, the $(x, y)$ components of its points were projected onto the surface of the sphere, and the resulting path followed for 2000 points (repeated for each of the five classes). At each of the 2000 views, Fourier magnitude 28-tuples were extracted. Time series were formed from the 1st, 14th, and 28th components of the resulting sequence of 2000 Fourier vectors. Grassberger and Procaccia analyses of each class revealed nearly linear log-log plots from which fractal dimensions could be estimated. See for example Fig. 8, in which the fourteenth Fourier component of the M60 sequence was examined. Based 


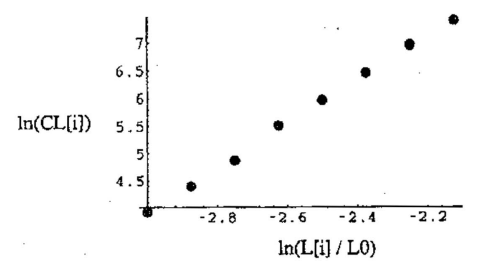

Fig. 8. $\quad G$ and $P$ plot for 14th Fourier component of M60.

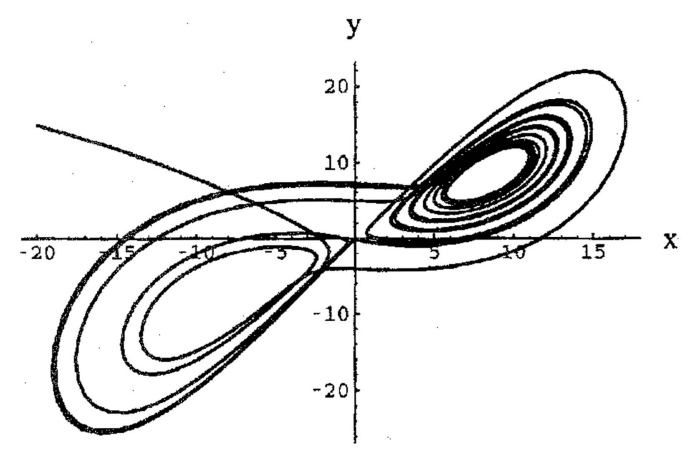

Fig. 9. Obtaining Lorenz test sequences.

on the slope of the line joining the endpoints of this data, the correlation dimension of the generating time series is estimated at about 3.96.

Similar investigations were performed for the $1 \mathrm{st}$ and 28th components of the M60 data, and for the $1 \mathrm{st}, 14 \mathrm{th}$, and 28th components of the remaining four military vehicles. The maximum fractal dimension so obtained was 5.25; this number was used to infer a minimum test sequence length of $11>2 \times 5.25$.

Test sequences were obtained near the Lorenz viewing trajectory, but not on it, by solving the Lorenz equations using a different initial condition than that used to generate the training trajectory. Fig. 9 shows the projected solution of the Lorenz equations when an initial condition of $\left(x_{0}=-20, y_{0}=15, z_{0}=15\right)$ was used. Ignoring the initial point, the next 20 points on this trajectory were used to construct test sequences of lengths 4, 8, and 11 (this allowed 17 sequences of length 4,13 of length 8 , and 10 of length 11 ). The 20th point is the first point after the knee of the initial portion of the trajectory; it is located at about $(x=-2, y=-4)$. Compare this trajectory to the trajectory used to derive the training data. This is illustrated in Fig. 3, where the initial condition used to determine the trajectory was $\left(x_{0}=5, y_{0}=5, z_{0}=\right.$ $5)$. Using the test sequences with all five vehicles, classification accuracy was $100 \%$ for sequences of lengths 11 and 8 , and $98 \%$ for sequences of length 4 . Thus sequences of length 11 proved sufficiently long to classify these particular test sequences perfectly; but so did sequences of length 8 , and even sequences of length 4 classified quite well.

There are several possible explanations for these results. First, the military vehicles may be so different that their training evolutions in $\Re^{28}$ overlap very little, or not at all. If this is the case, very short test

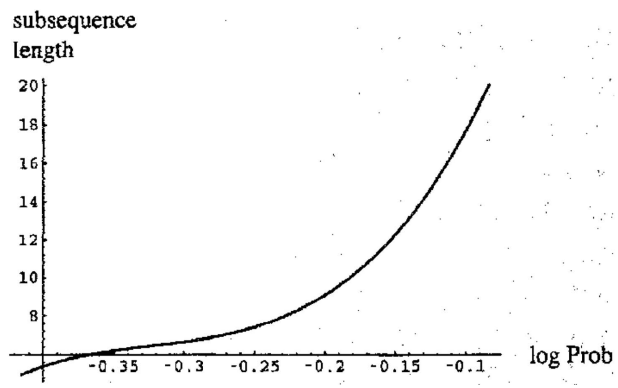

Fig. 10. Average log probabilities of M60 subsequences.

sequences will likely suffice for accurate classification. Second, there could be considerable overlap of evolutions, but the test sequences applied didn't fall near the regions of overlap. Third, the embedding theorem gives a sufficient, not necessary, sequence length to completely determine the evolution of an object. For example, eleven training sequence views of an M60 are sufficient to completely determine the evolution in $\Re^{28}$ of an M60; a number less than eleven may also suffice.

Test sequences were derived from Lorenz solutions using a couple of other initial conditions, with similar results. In both cases, using test sequences of length eleven yielded $100 \%$ classification accuracy, but often sequences of shorter length did the same.

Eleven views sufficed not only for synthetically generated views of the military vehicles, but also for actual video data recorded from a truck and a tank [5]. Using Hidden Markov Models trained using synthetic data, Fielding was able to classify with $100 \%$ accuracy the tank and truck, using in each case features extracted from only eleven frames of video.

Hidden Markov Model state transition information can be exploited to determine the probability that a given short sequence is representative of a longer sequence on which the model was trained [9]. Using as a time series the synthetic 14th Fourier component data for the M60 tank, and a Hidden Markov Model trained in leave-one-out fashion on that data, Fielding used subsequences ranging in length from 5 to 20 and calculated the average probabilities of subsequences of the various lengths, given the trained model. When the subsequence lengths were plotted against the logarithms of these probabilities, it was apparent that the probabilities increased with subsequence length; see Fig. 10. Consistent with the finding of eleven as a reasonable choice of number of views, it also appeared that subsequence lengths in the range of about 10 to 15 represent compromises between short sequence length and high probability of model identification.

\section{CONCLUSIONS}

The theory of embedded time series is applicable to the problem of determining a minimum test sequence length for spatio-temporal object recognition. 
Sequentially recorded feature vectors of a moving object form a training trajectory in feature space. Each of the sequences of feature vector components is a time series, and it is reasonable to expect each of these time series to have approximately the same fractal dimension if the feature space trajectory varies smoothly with viewing position. The embedding theorem may be applied to this fractal dimension to establish a sufficient number of observations to determine the feature space trajectory of the object. It was argued that this number is a reasonable lower bound on test sequence length for use in object classification. Experiments with data corresponding to five military vehicles (observed following a projected Lorenz trajectory on a viewing sphere) showed that this bound was indeed adequate. Exhaustive examination of all possible test sequences is not possible, and a shorter (or longer) test sequence length may be sufficient (or necessary) for certain classification tasks, depending on the similarity of the objects and the constraints on the test sequence trajectories. The technique described here provides a reasonable baseline.

\section{REFERENCES}

[1] Apostol, T. M. (1974)

Mathematical Analysis (2nd ed.).

Reading, MA: Addison-Wesley, 1974.

[2] Fielding, K. H., and Ruck, D. W.

Automatic object recognition using image sequences. AFIT TECH Report available from corresponding author.

[3] Fielding, K. H., Ruck, D. W., Rogers, S. K., Welsh, B. M., and Oxley, M. E. (1993)

Spatio-temporal pattern recognition using hidden Markov models.

Neural and Stochastic Methods in Image and Signal

Processing II Vol. 2032, July 1993, 144-154.
[4] Fielding, K. H., and Ruck, D. W.

Spatio-temporal pattern recognition using hidden Markov models.

IEEE Transactions on Aerospace and Electronic Systems, 31, 4 (Oct. 1995) 1292-1300.

[5] Fielding, K. H.

Spatial-temporal pattern recognition using hidden Markoy models.

$\mathrm{Ph} . \mathrm{D}$. dissertation, Air Force Institute of Technology, Wright-Patterson Air Force Base.

[6] Grassberger, P., and Procaccia, I. (1983)

Measuring the strangeness of strange attractors. Physica D, 9 (1983), 189-208.

[7] Hirsch, M. W., and Smale, S. (1974)

Differential Equations, Dynamical Systems, and Linear Algebra.

New York: Academic Press, 1974.

[8] Lorenz, E. N. (1963)

Deterministic nonperiodic flow.

Journal of the Atmospheric Sciences, 20 (1963), 130-141.

[9] Rabiner, L. R. (1989)

A tutorial on hidden Markov models and slelected applications in speech recognition. Proceedings of the IEEE, 77 (1989), 257-286.

[10] Sauer, T., et al. (1971)

Embedology.

Technical report 91-01-008, Santa Fe Institute, Santa Fe, NM, 1991.

[11] Seibert, M., and Waxman, A. M. (1992)

Adaptive 3-d object recognition from multiple views. IEEE Transactions on Pattern Analysis and Machine Intelligence, 12 (1992), 107-124. 
Capt. James R. Stright received a bachelor's degree in electrical engineering from Gannon University in Erie, PA, in 1984. In 1988 he received an M.S.E.E. degree from the Air Force Institute of Technology (AFIT), Wright-Patterson AFB.

His first Air Force assignment was to the National Computer Security Center at Fort Meade, MD, where he served as the government team leader for development of an encryption device. His next assignment was to Aeronautical Systems Division, Wright-Patterson Air Force Base, where he served as a computer security systems engineer on the Advanced Tactical Fighter program. Recieved Ph.D. in electrical engineering from AFIT in 1994. $\mathrm{He}$ is currently assigned to the Armament Directorate of Wright Laboratory at Eglin AFB, FL.

Capt. Stright is a member of SIAM.

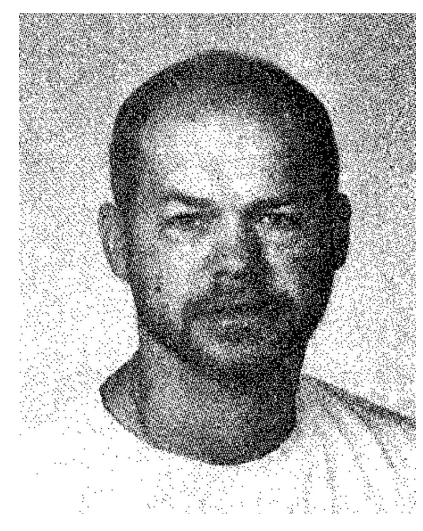

Steven K. Rogers is a Professor of Electrical Engineering in the Department of Electrical and Computer Engineering at the Air Force Institute of Technology, Wright-Patterson Air Force Base.

$\mathrm{He}$ is presently conducting an extensive research program on optical information processing and neural networks.

Dr. Rogers has published over 100 papers in the areas of neural networks, pattern recognition, and optical information processing. His textbook, Introduction to Biological and Artificial Neural Networks for Pattern Recognition, is being used around the world.

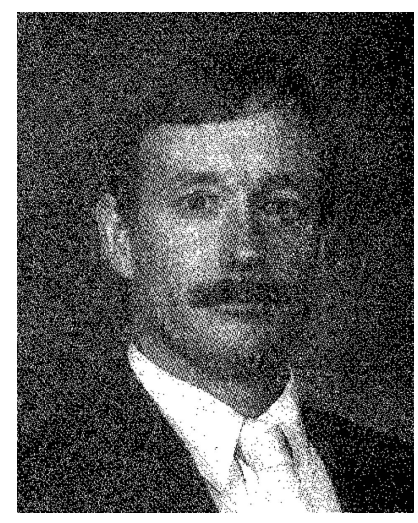

Dennis W. Quinn received his B.A. in mathematics in 1969 and his M.S. and Ph.D. degrees in applied mathematics in 1971 and 1973, respectively, from the University of Delaware, Newark.

He is currently a Professor of Mathematics in the Department of Mathematics and Statistics in the School of Engineering at the Air Force Institute of Technology (AFIT), Wright-Patterson Air Force Base. His research interests include air pollution modeling and physiology-based pharmacokinetic modeling. His publications deal with integral and finite element solutions of acoustic problems, using the method of characteristics in cancer risk assessment, the diffusion equation to model diffusion through the skin in pharmacokinetic modeling and the boundary element method for moving boundary problems.

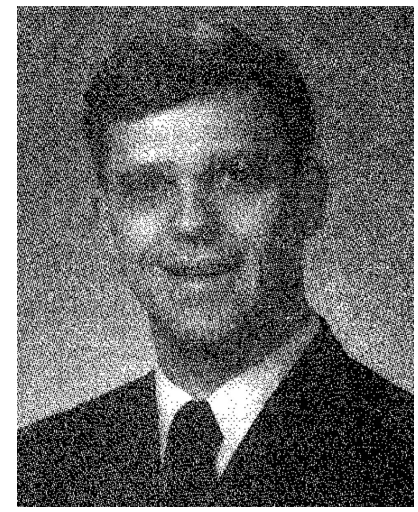

Capt. Kenneth H. Fielding received a BS in physics from the University of Central Arkansas in 1983. He entered the U.S. Air Force where he first attended the University of New Mexico, earning a BSEE degree in 1985. In 1988 he received an MSEE degree specializing in electro-optics from the Air Force Institute of Technology (AFIT), Wright-Patterson Air Force Base.

During 1985-1988, he was assigned to the Tactical Air Warfare Center for two years where he served as an electronic warfare engineer. His next assignment was to Rome Laboratory where, as chief electro-optics engineer, he performed research in optical automatic target recognition systems. He was awarded his Ph.D. in electrical engineering in 1994. He is currently assigned to Wright Laboratory at Wright-Patterson AFB, OH.

Capt. Fielding is a member of SPIE. 